\title{
Network of breathing. Multifunctional role of the diaphragm: a review. Response to the letter to Editor of Prof. Bordoni
}

The authors declare no financial disclosure

\section{Dear Editor,}

Thank you for the opportunity to respond to Professor Bordoni's letter. It is a great honor for us that our work has gained the interest of the scientist who in his research studies in a holistic way has explored the issues related to the main human respiratory muscle. In our work, we have focused on medical issues related to diaphragmatic function, i.e. its postural, cardiac, lymphatic, emesis, anti-reflux barrier and swallowing function. It was intended to show and emphasize other roles the muscle plays. The diaphragm is perceived primarily as the main respiratory muscle, but the clinical significance of its remaining functions is marginalized and undervalued.

The psychological side of the topic has been left to a separate review study. However, as rightly mentioned by Prof. Bordoni, the diaphragm has also properties related to the human emotional sphere, which was demonstrated in previous studies. First, emotional states very often alter the respiratory control. Dysfunctional breathing pattern is shown to be present in $11 \%$ of the general population, and among $83 \%$ of people suffering from anxiety. Fluoroscopic studies showed that in situations of tension and emotional stress, the diaphragm shows signs of hypertonicity by becoming flattened and immobile [1]. Secondly, the diaphragm has also postural functions and it should be taken into consideration that the po- sture is conditioned unequivocally by emotions and by the psyche. In sad situations, it comes to change our body posturę, i.e. the head is bent, the shoulders are internally rotated, and thoracic kyphosis is increased. The emotional states of grief, sorrow, worry, etc. lead to decrease the activity of the diaphragm and as a consequnce, the breathing becomes shorter and irregular and more controlled at the expense of the accessory muscle of respiration. Likewise, pleasurable emotional states such as happiness, pleasure tend to increase the diaphragm activity. Changes in posture when we are happy appear in the following way: raised head, shoulders turning outwards and physiological thoracic kyphosis with the diaphragm that has a marked movement, and in the ranges of the diaphragmatic movement and the breathing pattern, is fuller and more regular [2]. So, according to these studies, the diaphragm is responsive to the emotional states of its owner, as well as constitutes an accurate register of the character of the emotional states.

From clinical perspective, in this point it is necessary to cite the article of Prof. Bordoni about the impact of diaphragm on the threshold of pain. In this study the authors have decribed close relationship between the vagus and the phrenic nerves. The authors have suggested that dyssynergia between these two nerves could cause diaphragm pathological behavior and lead to non-respiratory symptoms (such as depression,

Address for correspondence: Janusz Kocjan, Chair and Department of Thoracic Surgery, Faculty of Medicine and Dentistry, Medical University of Silesia, Katowice, Poland, e-mail: j_kocjan@wp.pl

DOI: 10.5603/ARM.2017.0048

Received: 03.11.2017

Copyright (C) 2017 PTChP

ISSN 2451-4934 
anxiety and chronic pain), in patients with Chronic Obstructive Pulmonary Disease (COPD). An abnormal tension of the diaphragm in the region of the vagus nerve could cause a compression of the nerve, limiting its antinociceptive and anti-inflammatory ability. If the sympathetic nerves are compressed in the region of the diaphragm, their function and morphology can change, negatively affecting the innervated tissues [3].

To summarize, the diaphragm muscle has a broad repertoire of functions and plays an essential role in our physical, biochemical and emotional health. Due to its numerous links with other organs, we decided to use for the first time the term: „network of breathing”, to describe the range of diaphragm impact. We think that the letter of Prof. Bordoni and our answer to it constitute an important supplement to this article, describing the issue in a comprehensive manner. We also think that still it is little known about diaphragm function in existing literature. To fill the gap, further studies are undoubtedly needed. Emotional issues discussed here are certainly a prelude to the next review paper about the diaphragm.

\section{Conflict of interest}

The authors declare no conflict of interest.

\section{References:}

1. Courtney R. The functions of breathing and its dysfunctions and their relationship to breathing therapy. International Journal of Osteopathic Medicine. 2009; 12(3): 78-85, doi: 10.1016/j.ijosm.2009.04.002.

2. Armitage RB. The Law of Breathing and Chest Development. Society of Metaphysicians Ltd. 1993.

3. Bordoni B, Marelli F, Morabito B, et al. Depression, anxiety and chronic pain in patients with chronic obstructive pulmonary disease: the influence of breath. Monaldi Arch Chest Dis. 2017; 87(1): 811, doi: 10.4081/monaldi.2017.811, indexed in Pubmed: 28635197. 